\title{
CERN Neutrinos to Gran Sasso (CNGS): First Beam
}

\author{
Edda Gschwendtner on behalf of the CNGS Project and Commissioning Teams*
}

\begin{abstract}
The CNGS, CERN Neutrinos to Gran Sasso, project aims at directly detecting $v_{\mu}-v_{\tau}$ neutrino oscillations. An intense muon-neutrino beam $\left(10^{17} v_{\mu} /\right.$ day $)$ is generated at CERN and directed towards the Gran Sasso National Laboratory, LNGS, in Italy, $732 \mathrm{~km}$ away from CERN. In LNGS large and complex detectors will allow to detect, in particular, the rare tauneutrinos created by 'oscillation' from muon-neutrinos on their way between CERN and LNGS. On average around three tauneutrino events are predicted per year in each of the $\sim 2000$ ton detectors.

The construction of the CNGS beam facility started in September 2000, and the first neutrino beam has been produced in July 2006. In the presently approved physics programme, it is foreseen to run the facility for five years.
\end{abstract}

\section{INTRODUCTION}

A overview [1] of the CNGS neutrino beam facility at $\mathrm{A}_{\mathrm{CERN}}$ is shown in Fig. 1. During a CNGS cycle, i.e. every $6 \mathrm{~s}$, two SPS extractions of $2.4 \cdot 10^{13}$ protons each at $400 \mathrm{GeV} / \mathrm{c}$ are sent down the proton beam line to the target. The CNGS beam is extracted from the SPS using the same beam channel as for the LHC beam. At about $100 \mathrm{~m}$ from the extraction point, a string of switch magnet is used in order to transport the beam either to the LHC or to the CNGS target.

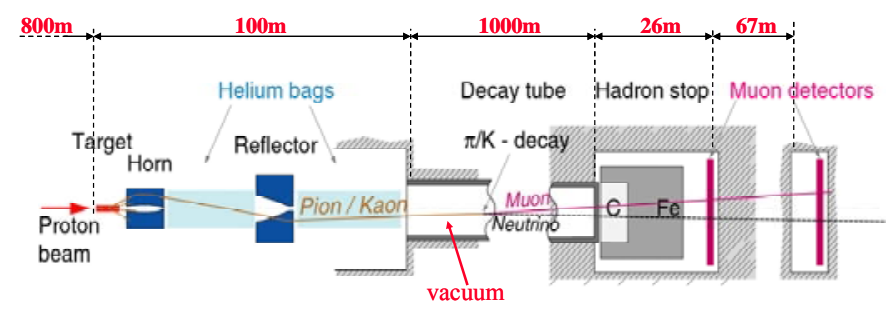

Fig. 1. CNGS layout.

In the graphite target, pions and kaons are produced (among many other particles). The positively charged $\pi / \mathrm{K}$ are energyselected and guided with two focusing lenses, i.e. horn and reflector, in the direction towards Gran Sasso. These particles decay in the $1000 \mathrm{~m}$ long decay vacuum tube into muonneutrinos and muons. The muons can be measured in the muon detectors. This allows concluding on the intensity of the neutrino beam produced and on the beam profile.

The muon-neutrino energy spectrum is the key feature for the experiments in Gran Sasso [2] as this determines the number of events to be measured. In order to maximize the number of tau-neutrino events in the detectors the product of two main parameters must match the neutrino spectrum of the CNGS beam (see Fig. 2): the oscillation probability between muonneutrino and tau-neutrino for a neutrino flight path of $732 \mathrm{~km}$

Manuscript received November 16, 2006.

E. Gschwendtner is with CERN, AB-Division, 1211 Geneva 23, Switzerland (telephone: +4122 76 77733, e-mail: edda.gschwendtner@cern.ch). and the production cross section of tau-neutrinos with matter, i.e. to leave a signal in the detector. The average muonneutrino energy is $17 \mathrm{GeV} / \mathrm{c}$.

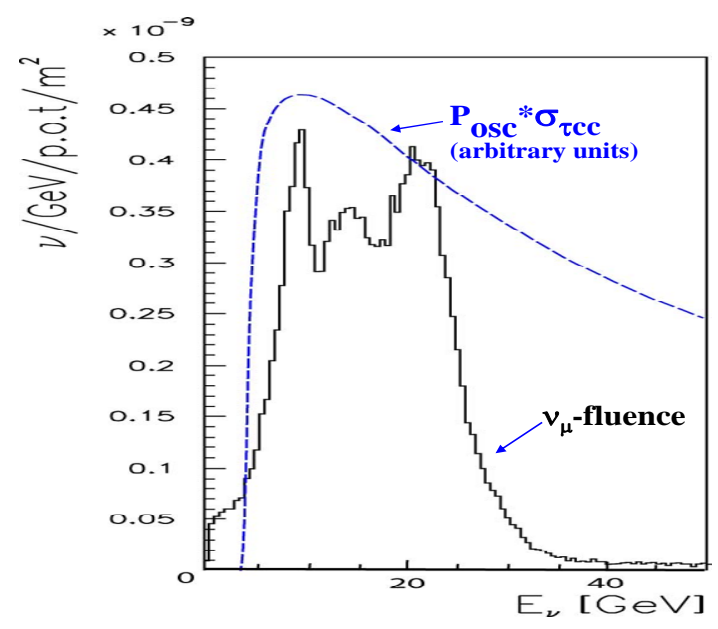

Fig. 2. Black curve: Monte-Carlo simulation of the muon-neutrino energy spectrum at Gran Sasso. Blue curve: the convolution of the probability for muon-neutrino to tau neutrino oscillation over $732 \mathrm{~km}$ and the interaction cross-section for tau-neutrinos in matter.

The civil construction work of the CNGS beam facility started in September 2000 and finished in 2004. In summer 2005 equipment installation in the proton beam line and the target chamber started; this work was completed in spring 2006.

During three weeks in July and August 2006 the primary beam line -from the SPS extraction to the target- and the secondary beam line -from the target to the muon monitorswas commissioned. In this context, the entire beam monitoring equipment has been tested and measurements have been compared with Monte Carlo simulations.

On $18^{\text {th }}$ August 2006 the CNGS facility started operation for physics, i.e. continuous production of neutrino beam for the experiments at Gran Sasso.

\section{PRIMARY BeAm Line COMMISSIONING}

The first proton beam was sent to the CNGS target on $11^{\text {th }}$ July 2006. The proton beam profile is measured using optical transition radiation screens. CCD cameras are used to observe the OTR radiation, a direct image of the proton beam crossing the screens. There are eight monitors along the $800 \mathrm{~m}$ long proton beam line. Already in the first shot the beam was found well centered in all of the eight screens (see Fig. 3).

The beam position monitors (BPMs), of which the buttons were recuperated from the Large Electron-Positron Collider, LEP, allow measuring the beam position very accurately. They revealed that the proton line was well tuned over its $800 \mathrm{~m}$ after only a minor magnetic correction. The maximum beam excursion measured is far less than the $+/-4 \mathrm{~mm}$ 
permitted from aperture constraints. Fig. 4 shows the horizontal and transversal beam trajectory along the proton beam line.

The beam size along the proton beam line was very close to the expectations from simulations; the beam spot at the target was found to be $0.5 \mathrm{~mm} \mathrm{r.m.s,} \mathrm{as} \mathrm{expected.} \mathrm{The} \mathrm{beam} \mathrm{position}$ stability onto the target has been averaged over several days and has been found to be $\sim 50 \mu \mathrm{m}$ r.m.s. This is much better than initially specified.
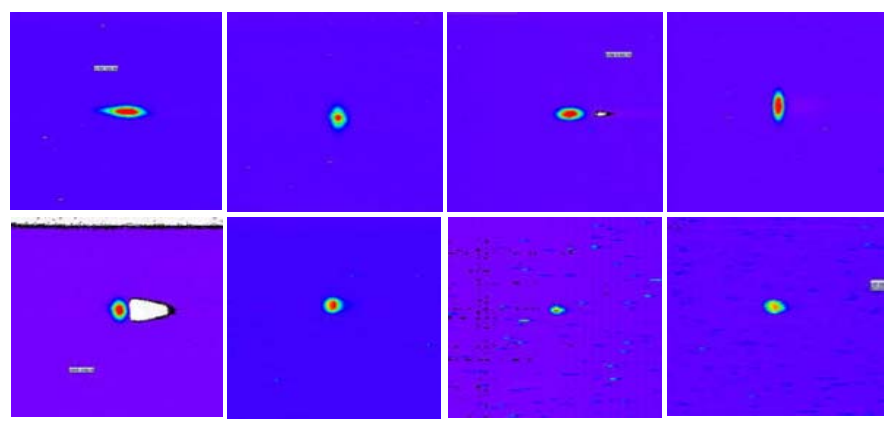

Fig. 3. Beam profiles of the first shot down the primary beam line measured with optical transition radiation screens. The total length of the beam line is $\sim 800 \mathrm{~m}$.

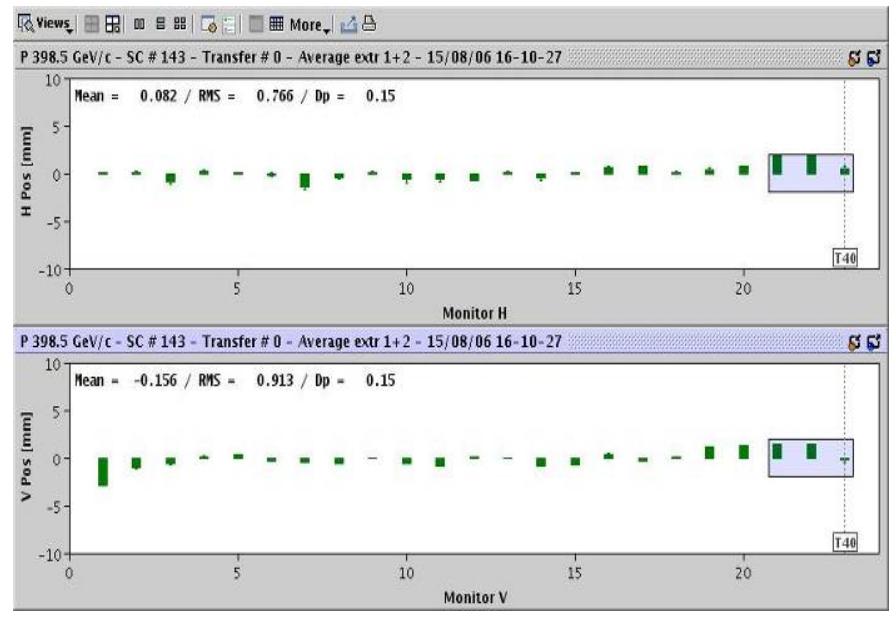

Fig. 4. Horizontal (top) and vertical (bottom) beam trajectory along the primary beam line. Each bar corresponds to a measurement in a beam position monitor.

\section{SECONDARY BeAm Line COMMISSIONING}

Fig. 5 shows the CNGS target magazine. The target unit consists of 13 graphite rods, each rod is $10 \mathrm{~cm}$ long and the rods are interspaced by $9 \mathrm{~cm}$. The diameter of the first two rods is $5 \mathrm{~mm}$; the other rods have a diameter of $4 \mathrm{~mm}$. The rods need to be thin and interspaced to let high-energy pions and kaons that are produced at smaller angles leave the target without re-interacting.

Five units are assembled into a target magazine (this is a rotating system to allow the change of a target unit). One unit will be used in beam; the other four are kept as spares.

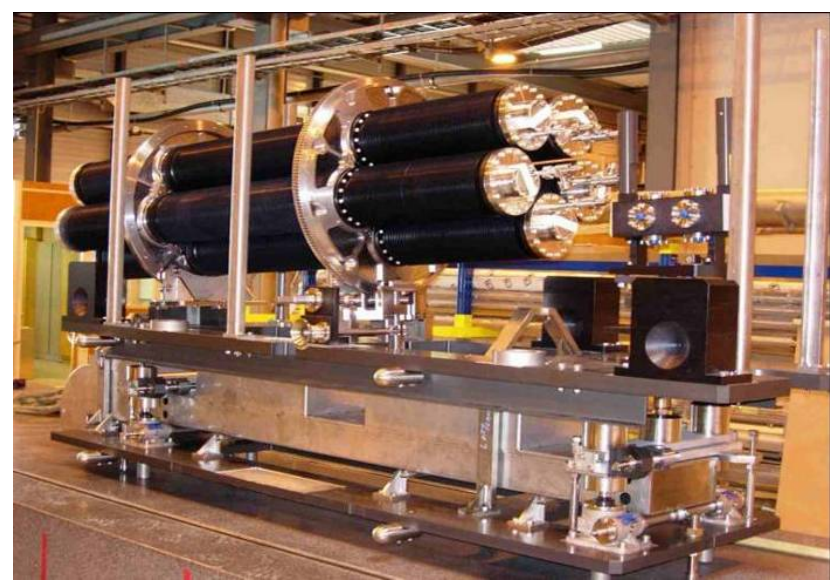

Fig. 5. CNGS target magazine consisting of five target units. Only the bottom unit is used, the other four are kept as spares.

The magnetic focusing system, comprising the horn and reflector, is installed downstream of the target. The horn and reflector are lenses with a focusing magnetic field created between the inner and outer conductor by a high, pulsed current. The shape of the inner conductor provides the desired focusing effect. Figure 6 shows the horn during installation. The horn is pulsed twice every $6 \mathrm{~s}$ cycle with a current of 150kA during a few milliseconds, the reflector with 180kA. The two pulses are separated by $50 \mathrm{~ms}$, in-time with the two beam pulses (see Fig. 7).

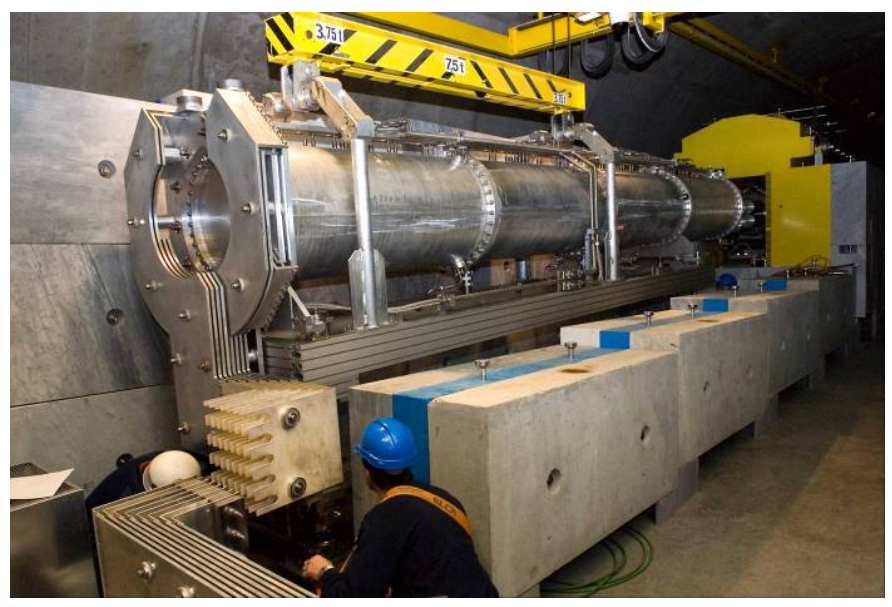

Fig. 6. The CNGS horn during installation.

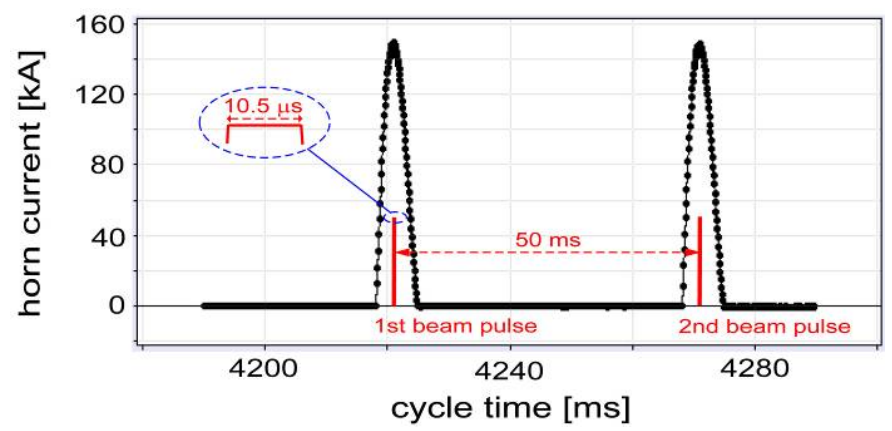

Fig. 7. Current pulses as measured in the CNGS horn (the same current pulses are observed for the reflector, but with a current of $180 \mathrm{kA}$ ). 
Special features are implemented to exchange the target or the horn remotely in case one of these systems should break down.

The centering of the proton beam on the target has been checked with the Target Beam Instrumentation Downstream, TBID monitor. In this detector secondary electrons are produced by charged particles traversing a $145 \mathrm{~mm}$ diameter, $12 \mu \mathrm{m}$ thick titanium sheet mounted in a vacuum box.

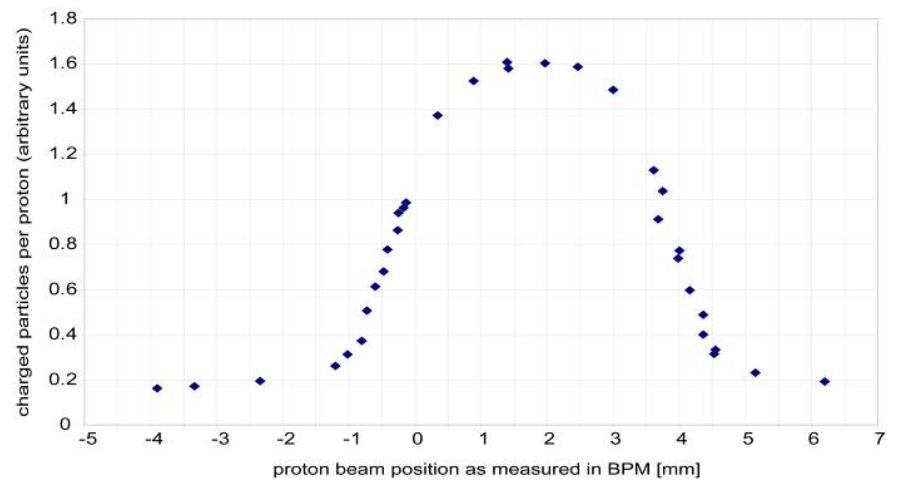

Fig. 8. The number of charged particles (arbitrary units) produced for different beam positions incident on the target. The proton beam should be positioned at $+2 \mathrm{~mm}$ to optimize the particle production in the target.

Since the TBID is installed immediately downstream of the target, a proton beam scan across the target provides information on the maximum production of charged particles, i.e. on the best alignment of the proton beam with respect to the target. The result of such a scan is shown in Fig. 8 - the observed signal in the TBID is a convolution of the target rod size and the proton beam spot.

One of the most important checks that can be performed in the neutrino beam line concerns the production of muons, created in association with the muon-neutrinos in the decay of the pions and kaons produced in the target.
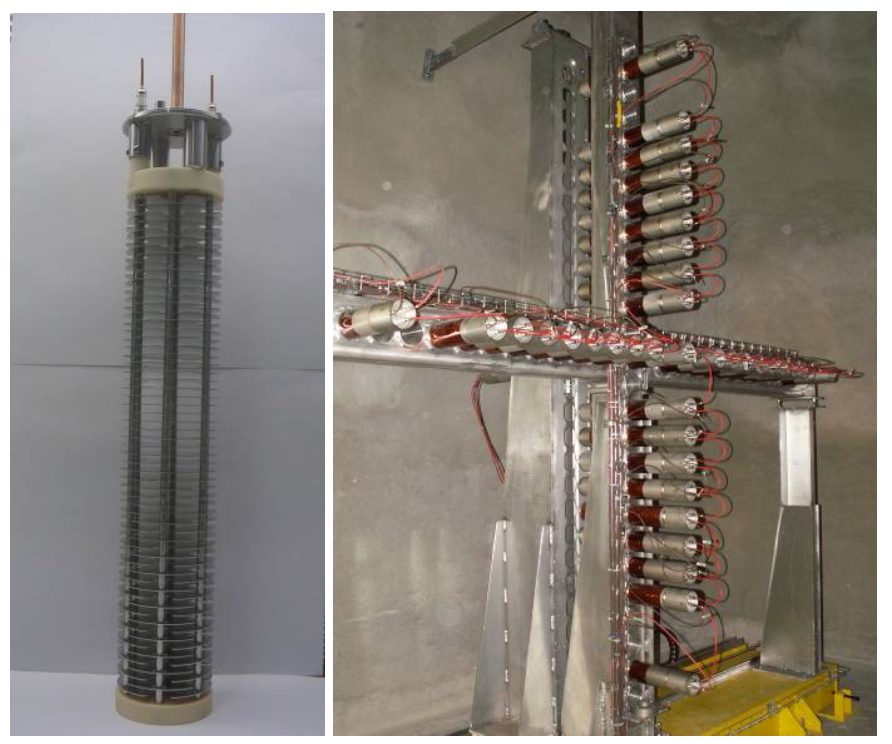

Fig. 9. Left: a single muon detector, without its stainless steel casing. Right: the muon detector cross-shaped array.
The two muon detector stations provide on-line feedback for the quality control of the neutrino beam.

These detectors must measure up to $10^{8}$ muons $/ \mathrm{cm}^{2}$ during one extraction, i.e. during $10.5 \mu$ s. To cope with such a high instantaneous rate we use nitrogen-filled, sealed ionization chambers. Each detector has 64 electrodes separated by $5 \mathrm{~mm}$. The electrode diameter is $4 \mathrm{~cm}$ and the bias voltage is set to 800 Volts. CNGS took advantage of the recent developments of ionization chambers at CERN, which will be used as beam loss monitors (BLMs) at the LHC [3]. The first 76 of more than 3000 of these BLMs are now in use at CNGS.

In each of the two CNGS muon detector chambers, there are 37 fixed muon detectors installed. They are assembled in a cross-shaped array (see Fig. 9) to provide the muon intensity and the vertical and horizontal muon profile. In addition an identical monitor is installed on a motorized support, downstream of the fixed ones to allow cross-calibration of the fixed monitors and to probe the muon profile where there is no fixed monitor.

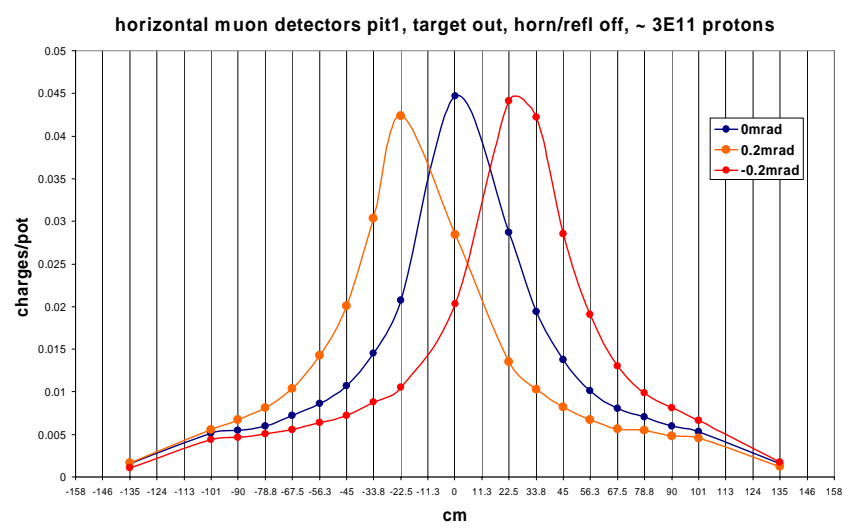

Fig. 10. Horizontal muon profile in the first muon detector chamber for different beam angles. The target is moved out. Each point corresponds to a detector and gives the induced charges per proton on target.

Fig. 10. shows the horizontal muon profile in the first muon detector chamber in a set of measurements without target and for different proton beam angles, i.e. $0 \mathrm{mrad}, 0.2 \mathrm{mrad}$ and $0.2 \mathrm{mrad}$. The muons observed here are mainly produced by proton induced reaction in the beam dump (hadron stop). We see that the muon detector signal is extremely sensitive to any angular misalignment. Similarly, using the muon monitor profile information (centroid, symmetry), we are able to optimize the alignment between the proton beam, the target and the horn/reflector.

The measurements are in reasonably good agreement with the preliminary expectations based on the FLUKA simulation package.

\section{FIRST CNGS OPERATION}

After CNGS has been successfully commissioned, the CNGS physics operation run started on $18^{\text {th }}$ August 2006 for two weeks.

A display providing online feedback for the quality of the neutrino beam is shown in Fig. 11, with both the horizontal 
and vertical muon profiles for the two muon detector chambers. Since the chambers are separated by $67 \mathrm{~m}$ of rock, the muon energy spectrum is different in the two chambers. Only muons with an energy above $20 \mathrm{GeV}(50 \mathrm{GeV})$ reach the first (second) muon detector chamber.

In Fig. 11 the muon profiles for both extractions are overlaid with different colors. There is hardly any difference between these two extractions, which indicates that the proton beam on target and the secondary beam equipment are very stable.

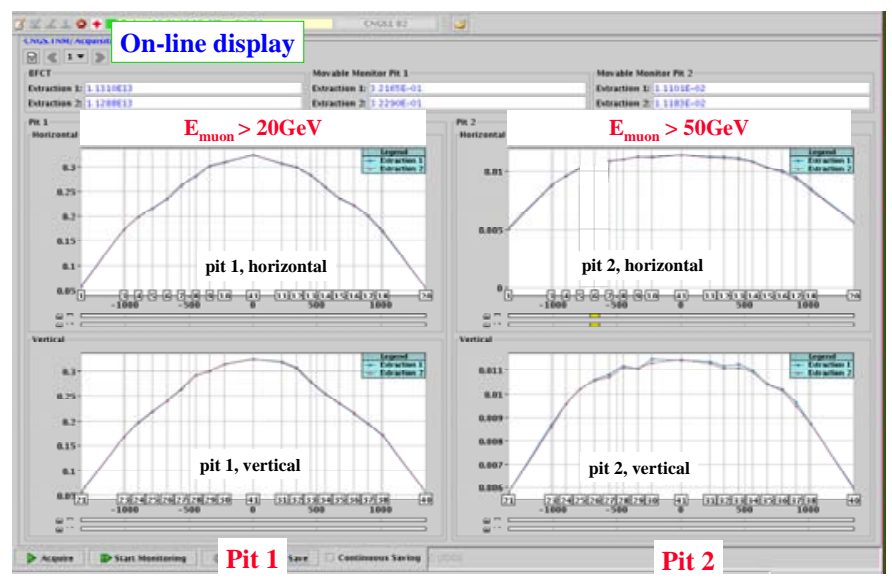

Fig. 11. Online display of the horizontal and vertical muon profiles in the first (pit 1) and second (pit 2) muon detector chamber.

\section{SUMMARY}

The CNGS construction started in 2000 and the installation was finished in beginning of 2006. A detailed hardware commissioning of the CNGS equipment (magnets, target, horn, etc...) has been performed. This was followed by the socalled 'dry-runs' of the CNGS facility, tests under the most realistic conditions -as if there were beam, but without beam. These 'dry-runs' allowed early debugging of all systems and saved a lot of beam time later.

By $18^{\text {th }}$ August 2006 the CNGS facility had been successfully commissioned.

The true challenge starts now, with continuous high intensity operation, where we will have to cope e.g. with high radiation levels in much of the CNGS area and fatigue effects on target, horn and reflector from the beam impact on the equipment.

\section{ACKNOWLEDGMENT}

Many thanks go to the equipment and control system experts, and to the CERN accelerator operations team, which all contributed to the success of CNGS. We also wish to thank our colleagues from Fermilab and KEK for the fruitful discussions during the design and construction of the CNGS facility.

\section{REFERENCES}

[1] 'General Description of the CNGS Project', 2000, http://projcngs.web.cern.ch/proj-cngs/

[2] Gran Sasso Laboratory: http://www.lngs.infn.it/, 'The OPERA Experiment', http://operaweb.web.cern.ch/operaweb/index.shtml

[3] M. Stockner et al, 'Measurements and Simulations of Ionization Chamber Signals in Mixed Radiation Fields for the LHC BLM System', NSS-IEEE06, San Diego, to be published, 2006.

\footnotetext{
* Members of the CNGS project and commissioning teams: G. Arduini, V. Baggiolini, T. Bogey, T. Bohl, L. Bruno, M. Clement, E. Chapochnikova, K. Cornelis, J.M. Cravero, B. Dehning, K. Elsener, GF Ferioli, F. Follin, D Forkel-Wirth, H. Gaillard, R. Giachino, B. Goddard, M. Gourber-Pace, W. Herr, D. Jacquet, R. Jones, L. Jensen, V. Kain, M. Lamont, L.A. Lopez Hernandez, G.. Maire, M. Meddahi, A. Pardons, V. Paris, L. Pereira, B. Pirollet, B. Puccio, S. Rangod, J. Ridewood, L. Rinolfi, G. Robin, S. Roesler, R. Schmidt, K. Sigerud, A. Spinks, N. Stapley, Heinz Vincke, Helmut Vincke, P. Voytila, J. Wenninger, M. Wilhelmsson.
} 